Bangladesh Journal of Bioethics 2012; 3(3):12-15

\title{
ISSUES OF RESEARCH ETHICS AND CLINICAL ETHICS IN DEVELOPING WORLD, POSSIBLE SOLUTION: A WAY FORWARD
}

\author{
Muhammad Waseem Khan ${ }^{1}$, Imrana Niaz Sultan ${ }^{2}$, Afrasiab Khan ${ }^{3}$ \\ Muhammad Bilal Khan ${ }^{4}$
}

Department of Biotechnology \& Informatics, Faculty of Life Sciences \& Informatics, Balochistan University of Information Technology Engineering \& Management Sciences (BUITEMS), Quetta Balochistan, Pakistan. Email: mwaseem.tareen@yahoo.com

\begin{abstract}
Research plays a pivotal role in the progress of inventions in medicines and medical technology. It is noticed that researchers from developing world or multinational companies are interested to conduct their research studies in developing countries, in doing so sometimes it is noticed that rather benefiting the participants it causes significant harm to the research study participants of developing countries. Ethical lapses in research can significantly harm human subjects. In research ethics the basic aim of ethics is distinguishing between right and wrong, to recognize the wrong doings and try to avoid them from harming research subjects in any research study whether that is done in developing country or developed country. It must follow the principle of non malefiecence or do no harm.
\end{abstract}

INTRODUCTION: There are many ethical issues regarding research ethics in developing countries that need to be addressed. The issue of study design, independent ethical review, valid inform consent, respect for individual's autonomy and dignity, social value of research, scientific validity of research, fair subject selection, risk benefits ratio, standards of care, socioeconomic deprivation inequities and post trial benefits to host community are largely have been highlighted but mostly ignored by researchers in developing countries. ${ }^{1,2}$

One central ethical concern when conducting research on human subjects in developing countries is to avoid research which has been rejected as unethical or of no value in the developed country. Many research studies that are conducted in developing countries are of no value and sometimes they are scientifically invalid, but still they are conducted by multinational companies in resource poor developing countries. These studies are largely conducted for financial gain rather than for the health care of human beings. It is noticed that such studies are conducted in areas where participants are heavily paid for their participation; the participants being very poor and illiterate tend to participate in large numbers regardless of their health as they receive enough amount to serve their needs. ${ }^{3}$

The issue of scientific validity and social value of any research study is important that lacks in developing countries research studies. Before conducting any research study the scientific validity must be ensured with the social value for the research participants, the primary beneficiaries of the research. The research study and design must be feasible within the social, political, and cultural context of the research participant's society. In developing countries it is noticed that the research studies are not carried out on the diseases which are causing more mortality and morbidity in developing countries rather it is largely conducted on the issues which are prevalent in the researcher's society from developed countries, which seems benefiting developed world and harming the participants from developing countries. The impact of research study needs to be addressed for the research participants rather than research conducting countries. ${ }^{4}$ 
A research study should advance scientific knowledge, should lead to improvements in health and should have social, scientific and clinical value. The research studies carried out in most developing countries are sometimes aimed for academic success, institutional demands, success and promotion and to secure research grants. The researchers tend to achieve relevant reports and results rather than improving the health of society. The researcher main aim should be the impact of research study for the improvement of health standards which must be above such kinds of selfish motives. ${ }^{5}$

The issue of involuntary participation is common in developing countries in which people from developing countries are forced to participate in any research study. Involuntary participation is linked with the issue of inform consent which is rather forced informed consent due to any reason. Inform consent means that research participants must be fully informed about the procedures, interventions and risks involved in research participation and must give their consent to participate. By complete consent it is meant that the five components of valid inform consent must be fulfilled which includes, disclosure (participant must be informed as fully as possible of the nature and purpose of the research, the procedures to be used, the expected risks and benefits to the participant/society, stresses, and discomforts, and alternatives to participating in the research), understanding (participant must understand what has been explained and must be given the opportunity to ask questions and have them answered by one of the investigators), voluntariness (participant's consent to participate in the research must be voluntary, free of any coercion or promises of benefits unlikely to result from participation), competence (participant must be competent to give consent. If the participant is not competent surrogate may provide consent if it is in the participant's best interest to participate), consent (potential human subject must authorize his/her participation in the research study). In developing countries Informed consent is considered just as singular event and a mere formality to be followed by researchers. The issue of standard of care is of great debate in recent years. In research studies in developing countries placebo has been used in control arms instead of universally accepted standard of care. It is mainly used in studies which are of no value to the society of research participants rather it is just for the registering of me too drugs and achieving financial gain and registering their products.

The issue of exposing vulnerable population is another issue that needs to be addressed in developing countries research studies. The study participants are not fairly selected and the procedure of randomization is not followed. Participants are recruited on subject of their easy availability which exposes vulnerable population to great risk and harm. Research ethics demands that study participants should be fairly selected without any biasness. Favorable risk-benefit ratio that determines the risks associated with conducting the trial should be clearly mentioned and the study participants should be given more benefit than the associated risks. ${ }^{4,6}$

Another important issue is the post trial benefits that should be focused and given importance especially once the research is over, pointing out that the new intervention proves to be effective and functioning then it should be offered to those in the control arm of the study subjects once the research is over. Research sponsored in developing countries by developed countries raises ethical issues not only during research but also once the research study is over. Researchers and sponsors should be bound by ethical committees to guarantee access to interventions to the research participants and to the local community when an intervention is found to be efficacious. The health care teams involved in research studies irrespective of being in develop or developing country is morally and ethically bound to respect human life, dignity and autonomy. They are morally bound to follow the principles of autonomy, beneficence by doing good, nonmaleficence by doing no harm to the research participants which requires investigators to minimize the harm and enhance benefits to the study participants and justice which requires priority to be given to the interests of research participants over his own.

HOW CAN CLINICAL RESEARCH BE MADE MORE ETHICAL IN DEVELOPING COUNTRIES? Clinical research also called clinical investigation is testing new drugs and compounds in human subjects for discovering potential beneficial effects. Clinical research is increasingly supported and 
conducted by multinational pharmaceutical companies. As clinical research involves patients, researchers are ethically and legally obligated to protect them. In clinical practice a physician would be expected to use interventions that have a reasonable expectation of success and are designed solely to enhance the well being of an individual patient.

Government hospitals and private hospitals in developing countries are aimed for better quality and affordable care to the masses. Patients in most developing countries choose public hospitals because they cannot afford treatment in private hospitals but even in public hospitals they pay for some drugs, tests and procedures, and this constitutes a burden that many cannot afford. Public hospitals in many developing countries are considered as clinical trial sites.

In a clinical setup of developing country the patients enters the trial because of their primary care physician is involved in research study. As physicians are highly respected and included by patients in their decisions in fact they are given the authority to make decisions for their patients. Patients are not able to question their doctor's judgment. They may be easily influenced by the doctor's advice. It is widely thought and believed that their refusal to follow their doctor's advice to enter a trial would affect their access to care. When the trial's principal investigator is also the patient's primary physician, there is scope for a direct conflict of interest. It becomes more suitable for conflict of interest when physicians are paid recruitment fees to recruit their patients into trials by the pharmaceutical companies conducting the research study. ${ }^{6}$

For any research to be ethical the ethics review committee has to play its role not to allow the research which has no impact, which is exploiting human population or causing harm to the participants. The ethics review committee should be independent bodies and the committees should be allowed to function in unbiased environment. The committee should focus the issues that are involved off the field and during the field when the study is conducted.

The physicians should be ethically and morally bound to protect his patient from harm and should not participate in trials which causes conflict of interest between his patient and the research, his primary aim should be his patient care rather than research. ${ }^{5}$

The physician participating in clinical research should be ethically and legally bound not to accept any gift, package and any benefit from pharmaceutical companies as it causes conflict of interest between the research and his patient care.

The issue of therapeutic misconception may be found in clinical research in developing countries where the patients are looking for cure while he is participating in research. The physician carrying the research should inform his patient before involving him in research. Being the patient's primary physician, he should protect him from risks regardless of valid inform consent which he must obtain from his patient. The physician should only advise his patient to enter in trial if it is in patient's benefits rather than receiving money for his participation.

The impact of research should be thoroughly analyzed and only those studies should be allowed by ethical reviews committees which have good impact for the society. It is noticed that many research studies conducted in developing countries are of no impact; they are only for financial gain, personal or degree and project requirements.

Another issue is of wild lab referrals and unnecessary lab procedures. In developing countries it is considered due to the physician having share in such procedures which may be demanded by pharmaceutical companies or lab owners. There should be double check on such procedures to ensure that poor patients are not exposed to expensive wild procedures.

The privacy and confidentiality is considered of no use in a clinical setup of many developing countries. Physicians and researchers must be sensitive to not only how information is protected from unauthorized observation, but also if and how participants are to be notified of any unforeseen findings from the research that they may or may not want to know. The investigator must enumerate how privacy and confidentiality concerns will be approached. 
CONCLUSION: Ethical guidelines and rules can be set easily but it is difficult to ensure that they are duly followed. We have to educate the inner human of individuals, the physicians and the researchers to act morally and ethically. We have to make it our habit and not to compromise on anything less than morality and virtuous values benefiting our fellow human beings.

\section{REFERENCES:}

1. Bhutta, Z, A. Ethics in international health research: a perspective from the developing world. Bulletin of the World Health Organization. 2002; 80:114-120.

2. Emanuel E J. et al. What Makes Clinical Research Ethical? JAMA. 2000; 283(20):2701-

2711.

3. Kiatboonsri P, Richter J. 'Unethical trials of dipyrone in Thailand'. Lancet, ii. 1988. 1491.

4. Emanuel EJ. et al. What Makes Clinical Research in Developing Countries Ethical?

The Bench marks of Ethical Research. Perspective. JID 2004 March 01:189.935.

5. Kalantri SP. Ethics in clinical research. Indian J. Anaesth. 2003; 47 (1): 30-32.

6. Benatar SR. Reflections and recommendations on research ethics in developing countries. Social Science \& Medicine. 54. 2002. 1131-1141.

Competing interests: The authors declare that they have no competing interests.

Authors' contributions: All authors have contributed substantially to the conception and design of the manuscript. Muhammad Waseem Khan is the $1^{\text {st }}$ and corresponding author. Imrana Niaz Sultan, Afrasiab Khan and Muhammad Bilal Khan are $2^{\text {nd }}$ authors and they have contributed equally. Muhammad Waseem Khan has critically revised the manuscript. All authors have read and approved the final manuscript.

Funding: The Authors declare that the current study was not funded. 